\title{
Multiplication theorems for multi-variable and multi-index Bessel functions
}

\author{
Ghazala Yasmin \\ Department of Mathematics, Aligarh Muslim University, Aligarh-202002, India \\ E-mail: ghazala30@gmail.com
}

\begin{abstract}
In this article, we derive multiplication theorems for the 3 -variable 2-parameter Bessel functions $J_{n}\left(\lambda x, \mu y, \nu z ; \tau_{1}, \tau_{2}\right)$ and 2-index 5-variable 3-parameter Bessel functions $J_{m, n}\left(\lambda x, \mu y, \nu z, \eta w, \beta h ; \tau_{1}, \tau_{2}, \tau_{3}\right)$ using the generating function method. Further, we derive multiplication theorems for functions related to $J_{n}\left(\lambda x, \mu y, \nu z ; \tau_{1}, \tau_{2}\right)$ and

$J_{m, n}\left(\lambda x, \mu y, \nu z, \eta w, \beta h ; \tau_{1}, \tau_{2}, \tau_{3}\right)$. Furthermore, we establish a multiplication theorem for N-index Bessel functions $J_{m_{1}, m_{2} \ldots . . m_{N}}(\lambda x)$.
\end{abstract}

Keywords: Multiplication theorem, Generalized Bessel functions, Double-index Bessel functions.

\section{Introduction}

Theory of special functions plays an important role in the formalism of mathematical physics. Bessel functions (BF) [13], are among the most important special functions, with very diverse applications to physics, engineering and mathematical physics ranging from abstract number theory and theoretical astronomy to concrete problems of physics and engineering.

Dattoli and his co-workers introduced and discussed various generalizations of BF within purely mathematical and applicative contexts (see for example [1-5,7,9-11]), as the success of any generalized special functions depend on its usefulness in applications and on the existence of effective numerical procedures for its computation. Generalized Bessel functions (GBF) have become a powerful tool to investigate the dynamical aspects of physical problems such as electron scattering by an intense linearly polarized laser wave, multi-photon processes and undulator radiation. The analytical and numerical study of GBF has revealed their interesting properties, which in some sense can be regarded as an extension of the properties of $\mathrm{BF}$ to a 2-dimensional domain. In this connection, the relevance of GBF and their multi-variable extension in mathematical physics has been emphasized, since they provide analytical solutions to partial differential equations such as the multi-dimensional diffusion equation, the Schrödinger and Klein-Gordon equations. A useful complement to the theory of GBF is offered by the introduction of 3-variable 2-parameter Bessel functions $(3 \mathrm{~V} 2 \mathrm{PBF}) J_{n}\left(x, y, z ; \tau_{1}, \tau_{2}\right)$ defined as ([8], p.222)

$$
J_{n}\left(x, y, z ; \tau_{1}, \tau_{2}\right)=\sum_{l=-\infty}^{\infty} \tau_{2}{ }^{l} J_{n-3 l}\left(x, y ; \tau_{1}\right) J_{l}(z), \quad 0<\left|\tau_{1}\right|,\left|\tau_{2}\right|<\infty
$$

where $\tau_{1}, \tau_{2}$ are arbitrary complex parameters. The generating function for $3 \mathrm{~V} 2 \mathrm{PBF}$ is given as

$$
\sum_{n=-\infty}^{\infty} J_{n}\left(x, y, z ; \tau_{1}, \tau_{2}\right) t^{n}=\exp \left(\frac{x}{2}\left(t-\frac{1}{t}\right)+\frac{y}{2}\left(t^{2} \tau_{1}-\frac{1}{t^{2} \tau_{1}}\right)+\frac{z}{2}\left(t^{3} \tau_{2}-\frac{1}{t^{3} \tau_{2}}\right)\right) .
$$

In particular, we note that

$$
J_{n}(x, y, z ; 1,1)=J_{n}(x, y, z),
$$


where $J_{n}(x, y, z)$ denotes the 3-variable generalized Bessel functions (3VGBF) defined by the generating function $[2]$

$$
\sum_{n=-\infty}^{\infty} J_{n}(x, y, z) t^{n}=\exp \left(\frac{x}{2}\left(t-\frac{1}{t}\right)+\frac{y}{2}\left(t^{2}-\frac{1}{t^{2}}\right)+\frac{z}{2}\left(t^{3}-\frac{1}{t^{3}}\right)\right) .
$$

Further, we note the following link

$$
J_{n}\left(x, y, 0 ; \tau_{1} \rightarrow \tau, 0\right)=J_{n}(x, y ; \tau)
$$

where $J_{n}(x, y ; \tau)$ denotes the 2-variable 1-parameter generalized Bessel functions (2V1PGBF) defined by the generating function [1]

$$
\sum_{n=-\infty}^{\infty} J_{n}(x, y ; \tau) t^{n}=\exp \left(\frac{x}{2}\left(t-\frac{1}{t}\right)+\frac{y}{2}\left(t^{2} \tau-\frac{1}{t^{2} \tau}\right)\right)
$$

and specified by the series

$$
J_{n}(x, y ; \tau)=\sum_{l=-\infty}^{\infty} \tau_{2}^{l} J_{n-2 l}(x) J_{l}(y) .
$$

Also, we note that

$$
J_{n}(x, y, 0 ; 1,1)=J_{n}(x, y),
$$

where $J_{n}(x, y)$ denotes the 2-variable generalized Bessel functions (2VGBF) defined by the generating function [6]

$$
\sum_{n=-\infty}^{\infty} J_{n}(x, y) t^{n}=\exp \left(\frac{x}{2}\left(t-\frac{1}{t}\right)+\frac{y}{2}\left(t^{2}-\frac{1}{t^{2}}\right)\right) .
$$

Moreover, it is evident that

$$
J_{n}(x, 0,0 ; 1,1)=J_{n}(x),
$$

where $J_{n}(x)$ denotes the ordinary Bessel functions (BF) defined by the generating function [13]

$$
\sum_{n=-\infty}^{\infty} J_{n}(x) t^{n}=\exp \left(\frac{x}{2}\left(t-\frac{1}{t}\right)\right)
$$

We considered the 2-index 5-variable 3-parameter Bessel functions (2I5V3PBF) $J_{m, n}\left(x, y, z, w, h ; \tau_{1}, \tau_{2}, \tau_{3}\right)$ defined as

$$
J_{m, n}\left(x, y, z, w, h ; \tau_{1}, \tau_{2}, \tau_{3}\right)=\sum_{l=-\infty}^{\infty} \tau_{3}^{l} J_{m-l}\left(x, z ; \tau_{1}\right) J_{n-l}\left(y, w ; \tau_{2}\right) J_{l}(h),
$$

with the following generating function

$$
\begin{gathered}
\sum_{m, n=-\infty}^{\infty} J_{m, n}\left(x, y, z, w, h ; \tau_{1}, \tau_{2}, \tau_{3}\right) u^{m} v^{n}=\exp \left(\frac{x}{2}\left(u-\frac{1}{u}\right)+\frac{y}{2}\left(v-\frac{1}{v}\right)+\frac{z}{2}\left(u^{2} \tau_{1}-\frac{1}{u^{2} \tau_{1}}\right)\right. \\
+\frac{w}{2}\left(v^{2} \tau_{2}-\frac{1}{v^{2} \tau_{2}}\right)+\frac{h}{2}\left(u v \tau_{3}-\frac{1}{u v \tau_{3}}\right) .
\end{gathered}
$$

In particular, we note that

$$
J_{m, n}(x, y, z, w, h ; 1,1,1)=J_{m, n}(x, y, z, w, h),
$$


where $J_{m, n}(x, y, z, w, h)$ denotes the 2-index 5 -variable Bessel functions (2I5VBF) defined by the generating function $([6]$, p.188(3.23))

$\sum_{m, n=-\infty}^{\infty} J_{m, n}(x, y, z, w, h) u^{m} v^{n}=\exp \left(\frac{x}{2}\left(u-\frac{1}{u}\right)+\frac{y}{2}\left(v-\frac{1}{v}\right)+\frac{z}{2}\left(u^{2}-\frac{1}{u^{2}}\right)+\frac{w}{2}\left(v^{2}-\frac{1}{v^{2}}\right)+\frac{h}{2}\left(u v-\frac{1}{u v}\right)\right)$.

Further, we note the following link

$$
J_{m, n}\left(x, y, 0,0, h \rightarrow z ; \tau_{1}, \tau_{2}, \tau_{3} \rightarrow \tau\right)=J_{m, n}(x, y, z ; \tau),
$$

where $J_{m, n}(x, y, z ; \tau)$ denotes the 2-index 3-variable 1-parameter Bessel functions (2I3V1PBF) defined by the generating function $([10]$, p.344(2))

$$
\sum_{m, n=-\infty}^{\infty} J_{m, n}(x, y, z ; \tau) u^{m} v^{n}=\exp \left(\frac{x}{2}\left(u-\frac{1}{u}\right)+\frac{y}{2}\left(v-\frac{1}{v}\right)+\frac{z}{2}\left(u v \tau-\frac{1}{u v \tau}\right)\right) .
$$

Furthermore, it is evident that

$$
J_{m, n}\left(x, y, 0,0, h \rightarrow z ; \tau_{1}, \tau_{2}, 1\right)=J_{m, n}(x, y, z),
$$

where $J_{m, n}(x, y, z)$ denotes the 2-index 3-variable Bessel functions (2I3VBF) defined by the generating function ([7], p.3639(13))

$$
\sum_{m, n=-\infty}^{\infty} J_{m, n}(x, y, z) u^{m} v^{n}=\exp \left(\frac{x}{2}\left(u-\frac{1}{u}\right)+\frac{y}{2}\left(v-\frac{1}{v}\right)+\frac{z}{2}\left(u v-\frac{1}{u v}\right)\right) .
$$

Also, we note that

$$
J_{m, n}\left(x, y \rightarrow x, 0,0, h \rightarrow x ; \tau_{1}, \tau_{2}, 1\right)=J_{m, n}(x),
$$

where $J_{m, n}(x)$ denotes the 2-index Bessel functions (2IBF) defined by the generating function ([7], p.3637(1))

$$
\sum_{m, n=-\infty}^{\infty} J_{m, n}(x) u^{m} v^{n}=\exp \left(\frac{x}{2}\left(\left(u-\frac{1}{u}\right)+\left(v-\frac{1}{v}\right)+\left(u v-\frac{1}{u v}\right)\right)\right) .
$$

Also, the 2-index 1-parameter Bessel functions $(2 \mathrm{I} 1 \mathrm{PBF}) J_{m, n}(x ; \tau)$ defined by means of the series [7]

$$
J_{m, n}(x ; \tau)=\sum_{l=-\infty}^{\infty} \tau^{l} J_{m-l}(x) J_{n-l}(x) J_{l}(x)
$$

Moreover, we note that

$$
J_{m, n}\left(x, 0, z \rightarrow y, 0,0 ; 1, \tau_{2}, \tau_{3}\right)=J_{m}(x, y),
$$

or

$$
J_{m, n}\left(0, y \rightarrow x, 0, w \rightarrow y, 0 ; \tau_{1}, 1, \tau_{3}\right)=J_{n}(x, y)
$$

and

$$
J_{m, n}\left(x, 0,0,0,0 ; \tau_{1}, \tau_{2}, \tau_{3}\right)=J_{m}(x),
$$

or

$$
J_{m, n}\left(0, y \rightarrow x, 0,0,0 ; \tau_{1}, \tau_{2}, \tau_{3}\right)=J_{n}(x)
$$

Next, we recall the 3 -variable generalized Hermite polynomials (3VGHP) $H_{n}(x, y, z)$ defined by the generating function $([3]$, p.511(17))

$$
\sum_{n=0}^{\infty} H_{n}(x, y, z) \frac{t^{n}}{n !}=\exp \left(x t+y t^{2}+z t^{3}\right)
$$

which for $z=0$ reduce to the $2 \mathrm{VHKdFP} H_{n}(x, y)$ defined by the generating function [5]

$$
\sum_{n=0}^{\infty} H_{n}(x, y) \frac{t^{n}}{n !}=\exp \left(x t+y t^{2}\right)
$$


and specified by the series

$$
H_{n}(x, y)=n ! \sum_{m=0}^{\left[\frac{n}{2}\right]} \frac{(-y)^{m}(2 x)^{n-2 m}}{m !(n-2 m) !} .
$$

The 2-index 5 -variable generalized Hermite polynomials (2I5VGHP) $H_{m, n}(x, y, z, w, h)$ defined by the generating function $[6]$

$$
\sum_{m, n=0}^{\infty} H_{m, n}(x, y, z, w, h) \frac{u^{m} v^{n}}{m ! n !}=\exp \left(x u+y v+z u^{2}+w v^{2}+h u v\right)
$$

which for $w=h=0$ reduce to the 2-index 3 -variable Hermite polynomials (2I3VHP) $H_{m, n}(x, y, z)$ defined by [14]

$$
H_{m, n}(x, y, z)=\sum_{p=0}^{m i n(m, n)} \frac{\left(\frac{x}{2}\right)^{m-p}\left(\frac{y}{2}\right)^{n-p}\left(\frac{z}{2}\right)^{p} m ! n !}{p !(m-p) !(n-p) !} .
$$

Further, the 2-index Hermite polynomials (2IHP) $H_{m, n}(x)$ are specified by the series [5]

$$
H_{m, n}(x)=\sum_{p=0}^{\min (m, n)} \frac{\left(\frac{x}{2}\right)^{m+n-p} m ! n !}{p !(m-p) !(n-p) !} .
$$

Hermite polynomials are useful complements to the theory of generalized Bessel functions. The link between these two families can be easily expressed as follows:

$$
J_{n}(x, y, z ; 1,1)=\sum_{r=0}^{\infty} \frac{1}{r !(n+r) !} H_{n+r}\left(\frac{x}{2}, \frac{y}{2}, \frac{z}{2}\right) H_{r}\left(\frac{-x}{2}, \frac{-y}{2}, \frac{-z}{2}\right)
$$

and

$$
J_{m, n}(x, y, z, w, h ; 1,1,1)=\sum_{r, s=0}^{\infty} \frac{1}{r ! s !(m+r) !(n+s) !} H_{m+r, n+s}\left(\frac{x}{2}, \frac{y}{2}, \frac{z}{2}, \frac{w}{2}, \frac{h}{2}\right) H_{r, s}\left(\frac{-x}{2}, \frac{-y}{2}, \frac{-z}{2}, \frac{-w}{2}, \frac{-h}{2}\right) .
$$

Theory of generalized Bessel functions has been developed mainly for their practical importance in many physical and engineering problems, ranging from non-dipolar scattering to surface diffraction theory. Motivated by the importance of multivariable forms of Bessel functions and their link with other forms of generalized Bessel functions, in this paper we establish multiplication theorems for the $3 \mathrm{~V} 2 \mathrm{PBF} J_{n}\left(\lambda x, \mu y, \nu z ; \tau_{1}, \tau_{2}\right)$ and $2 \mathrm{I} 5 \mathrm{~V} 3 \mathrm{PBF}$ $J_{m, n}\left(\lambda x, \mu y, \nu z, \eta w, \beta h ; \tau_{1}, \tau_{2}, \tau_{3}\right)$ using the generating function method. Also,we derive multiplication theorems for functions related to $J_{n}\left(\lambda x, \mu y, \nu z ; \tau_{1}, \tau_{2}\right)$ and $J_{m, n}\left(\lambda x, \mu y, \nu z, \eta w, \beta h ; \tau_{1}, \tau_{2}, \tau_{3}\right)$ as applications. Finally, we prove a multiplication theorem for N-index Bessel functions (N-IBF) $J_{m_{1}, m_{2} \ldots . . m_{N}}(\lambda x)$ as a possible extension of our method.

In section 2 , we derive multiplication theorems for the $3 \mathrm{~V} 2 \mathrm{PBF} J_{n}\left(\lambda x, \mu y, \nu z ; \tau_{1}, \tau_{2}\right) 2 \mathrm{I} 5 \mathrm{~V} 3 \mathrm{PBF}$

$J_{m, n}\left(\lambda x, \mu y, \nu z, \eta w, \beta h ; \tau_{1}, \tau_{2}, \tau_{3}\right)$. In section 3 , we consider applications of our main results. In section 4, concluding remarks are given.

\section{Multiplication theorems for 3V2PBF $J_{n}\left(\lambda x, \mu y, \nu z ; \tau_{1}, \tau_{2}\right)$ and 2I5V3PBF $J_{m, n}\left(\lambda x, \mu y, \nu z, \eta w, \beta h ; \tau_{1}, \tau_{2}, \tau_{3}\right)$}

In this section, we prove the following multiplication theorems involving $3 \mathrm{~V} 2 \mathrm{PBF} J_{n}\left(\lambda x, \mu y, \nu z ; \tau_{1}, \tau_{2}\right)$ and $2 \mathrm{I} 5 \mathrm{~V} 3 \mathrm{PBF}$ $J_{m, n}\left(\lambda x, \mu y, \nu z, \eta w, \beta h ; \tau_{1}, \tau_{2}, \tau_{3}\right)$ :

Theorem 2.1 The following multiplication theorem for the 3V2PBF $J_{n}\left(\lambda x, \mu y, \nu z ; \tau_{1}, \tau_{2}\right)$ holds true:

$$
J_{n}\left(\lambda x, \mu y, \nu z ; \tau_{1}, \tau_{2}\right)=\lambda^{-n} \sum_{m=0}^{\infty} \frac{1}{m !} J_{n-m}\left(x, y, z ; \frac{\lambda^{2} \tau_{1}}{\mu}, \frac{\lambda^{3} \tau_{2}}{\nu}\right) H_{m}\left(\frac{x}{2}\left(\lambda^{2}-1\right), \frac{\lambda^{2} \tau_{1} y}{2 \mu}\left(\mu^{2}-1\right), \frac{\lambda^{3} \tau_{2} z}{2 \nu}\left(\nu^{2}-1\right)\right) .
$$


Proof We start with the identity

$$
\begin{aligned}
& \exp \left(\frac{\lambda x}{2}\left(t-\frac{1}{t}\right)+\frac{\mu y}{2}\left(t^{2} \tau_{1}-\frac{1}{t^{2} \tau_{1}}\right)+\frac{\nu z}{2}\left(t^{3} \tau_{2}-\frac{1}{t^{3} \tau_{2}}\right)\right) \\
& =\exp \left(\frac{x}{2}\left(\frac{t}{\lambda}-\frac{\lambda}{t}\right)\right) \exp \left(\frac{y}{2}\left(\frac{t^{2} \tau_{1}}{\mu}-\frac{\mu}{t^{2} \tau_{1}}\right)\right) \exp \left(\frac{z}{2}\left(\frac{t^{3} \tau_{2}}{\nu}-\frac{\nu}{t^{3} \tau_{2}}\right)\right) \\
& \exp \left(\frac{x}{2}\left(\lambda^{2}-1\right)\left(\frac{t}{\lambda}\right)+\frac{\lambda^{2} \tau_{1} y}{2 \mu}\left(\mu^{2}-1\right)\left(\frac{t}{\lambda}\right)^{2}+\frac{\lambda^{3} \tau_{2} z}{2 \nu}\left(\nu^{2}-1\right)\left(\frac{t}{\lambda}\right)^{3}\right)
\end{aligned}
$$

which on using the definitions (11) and (27), on the r.h.s. becomes

$$
\begin{gathered}
\exp \left(\frac{\lambda x}{2}\left(t-\frac{1}{t}\right)+\frac{\mu y}{2}\left(t^{2} \tau_{1}-\frac{1}{t^{2} \tau_{1}}\right)+\frac{\nu z}{2}\left(t^{3} \tau_{2}-\frac{1}{t^{3} \tau_{2}}\right)\right) \\
=\sum_{h, k, p=-\infty}^{\infty} \frac{1}{\lambda^{h}} J_{h}(x)\left(\frac{\tau_{1}}{\mu}\right)^{k}\left(\frac{\tau_{2}}{\nu}\right)^{p} t^{h+2 k+3 p} J_{k}(y) J_{p}(z) \sum_{m=0}^{\infty}\left(\frac{t}{\lambda}\right)^{m} \frac{1}{m !} \\
H_{m}\left(\frac{x}{2}\left(\lambda^{2}-1\right), \frac{\lambda^{2} \tau_{1} y}{2 \mu}\left(\mu^{2}-1\right), \frac{\lambda^{3} \tau_{2} z}{2 \nu}\left(\nu^{2}-1\right)\right) .
\end{gathered}
$$

Now, replacing $h$ by $s-2 k$ and using the relation (7) in the r.h.s. of Eq.(37), we find

$$
\begin{gathered}
\exp \left(\frac{\lambda x}{2}\left(t-\frac{1}{t}\right)+\frac{\mu y}{2}\left(t^{2} \tau_{1}-\frac{1}{t^{2} \tau_{1}}\right)+\frac{\nu z}{2}\left(t^{3} \tau_{2}-\frac{1}{t^{3} \tau_{2}}\right)\right) \\
=\sum_{s, p=-\infty}^{\infty} \frac{1}{\lambda^{s}} J_{s}\left(x, y ; \frac{\lambda^{2} \tau_{1}}{\mu}\right)\left(\frac{\tau_{2}}{\nu}\right)^{p} t^{s+3 p} J_{p}(z) \sum_{m=0}^{\infty}\left(\frac{t}{\lambda}\right)^{m} \frac{1}{m !} \\
H_{m}\left(\frac{x}{2}\left(\lambda^{2}-1\right), \frac{\lambda^{2} \tau_{1} y}{2 \mu}\left(\mu^{2}-1\right), \frac{\lambda^{3} \tau_{2} z}{2 \nu}\left(\nu^{2}-1\right)\right) .
\end{gathered}
$$

Again, replacing $s$ by $q-3 p$ and using the relation (1) in the r.h.s. of Eq.(38), we get 


$$
\begin{array}{r}
\exp \left(\frac{\lambda x}{2}\left(t-\frac{1}{t}\right)+\frac{\mu y}{2}\left(t^{2} \tau_{1}-\frac{1}{t^{2} \tau_{1}}\right)+\frac{\nu z}{2}\left(t^{3} \tau_{2}-\frac{1}{t^{3} \tau_{2}}\right)\right) \\
=\sum_{q=-\infty}^{\infty} \sum_{m=0}^{\infty}\left(\frac{t}{\lambda}\right)^{q+m} \frac{1}{m !} J_{q}\left(x, y, z ; \frac{\lambda^{2} \tau_{1}}{\mu}, \frac{\lambda^{3} \tau_{2}}{\nu}\right) \\
H_{m}\left(\frac{x}{2}\left(\lambda^{2}-1\right), \frac{\lambda^{2} \tau_{1} y}{2 \mu}\left(\mu^{2}-1\right), \frac{\lambda^{3} \tau_{2} z}{2 \nu}\left(\nu^{2}-1\right)\right) .
\end{array}
$$

Further, replacing $q$ by $n-m$ in the r.h.s. and using definition (2) on the l.h.s. of above equation, we find

$$
\begin{gathered}
\sum_{n=-\infty}^{\infty} J_{n}\left(\lambda x, \mu y, \nu z ; \tau_{1}, \tau_{2}\right) t^{n}=\sum_{n=-\infty}^{\infty} t^{n} \lambda^{-n} \sum_{m=0}^{\infty} \frac{1}{m !} J_{n-m}\left(x, y, z ; \frac{\lambda^{2} \tau_{1}}{\mu}, \frac{\lambda^{3} \tau_{2}}{\nu}\right) \\
H_{m}\left(\frac{x}{2}\left(\lambda^{2}-1\right), \frac{\lambda^{2} \tau_{1} y}{2 \mu}\left(\mu^{2}-1\right), \frac{\lambda^{3} \tau_{2} z}{2 \nu}\left(\nu^{2}-1\right)\right) .
\end{gathered}
$$

Finally, on equating the coefficients of like powers of $t$ in Eq. (40), we get the assertion (35) of Theorem 2.1.

Theorem 2.2 The following multiplication theorem for the $2 I 5 \mathrm{~V} 3 \mathrm{PBF} J_{m, n}\left(\lambda x, \mu y, \nu z, \eta w, \beta h ; \tau_{1}, \tau_{2}, \tau_{3}\right)$ holds true:

$$
\begin{gathered}
J_{m, n}\left(\lambda x, \mu y, \nu z, \eta w, \beta h ; \tau_{1}, \tau_{2}, \tau_{3}\right)=\lambda^{-m} \mu^{-n} \sum_{i, j=0}^{\infty} \frac{\lambda^{i} \mu^{j}}{i ! j !} J_{m-i, n-j}\left(x, y, z, w, h ; \frac{\lambda^{2} \tau_{1}}{\nu}, \frac{\mu^{2} \tau_{2}}{\eta}, \frac{\lambda \mu \tau_{3}}{\beta}\right) \\
H_{i, j}\left(\frac{x}{2 \lambda}\left(\lambda^{2}-1\right), \frac{y}{2 \mu}\left(\mu^{2}-1\right), \frac{z \tau_{1}}{2 \nu}\left(\nu^{2}-1\right), \frac{w \tau_{2}}{2 \eta}\left(\eta^{2}-1\right), \frac{h \tau_{3}}{2 \beta}\left(\beta^{2}-1\right)\right) .
\end{gathered}
$$

Proof We start with the identity

$$
\begin{gathered}
\exp \left(\frac{\lambda x}{2}\left(u-\frac{1}{u}\right)+\frac{\mu y}{2}\left(v-\frac{1}{v}\right)+\frac{\nu z}{2}\left(u^{2} \tau_{1}-\frac{1}{u^{2} \tau_{1}}\right)+\frac{\eta w}{2}\left(v^{2} \tau_{2}-\frac{1}{v^{2} \tau_{2}}\right)+\frac{\beta h}{2}\left(u v \tau_{3}-\frac{1}{u v \tau_{3}}\right)\right) \\
=\exp \left(\frac{x}{2}\left(\frac{u}{\lambda}-\frac{\lambda}{u}\right)\right) \exp \left(\frac{y}{2}\left(\frac{v}{\mu}-\frac{\mu}{v}\right)\right) \exp \left(\frac{z}{2}\left(\frac{u^{2} \tau_{1}}{\nu}-\frac{\nu}{u^{2} \tau_{1}}\right)\right) \exp \left(\frac{w}{2}\left(\frac{v^{2} \tau_{2}}{\eta}-\frac{\eta}{v^{2} \tau_{2}}\right)\right) \\
\exp \left(\frac{h}{2}\left(\frac{u v \tau_{3}}{\beta}-\frac{\beta}{u v \tau_{3}}\right)\right) \exp \left(\frac{x}{2}\left(\frac{\lambda^{2}-1}{\lambda}\right) u+\right. \\
\left.\frac{y}{2}\left(\frac{\mu^{2}-1}{\mu}\right) v+\frac{z \tau_{1}}{2}\left(\frac{\nu^{2}-1}{\nu}\right) u^{2}+\frac{w \tau_{2}}{2}\left(\frac{\eta^{2}-1}{\eta}\right) v^{2}+\frac{h \tau_{3}}{2}\left(\frac{\beta^{2}-1}{\beta}\right) u v\right),
\end{gathered}
$$

which on using the definitions (11) and (30), on the r.h.s. becomes

$$
\begin{gathered}
\exp \left(\frac{\lambda x}{2}\left(u-\frac{1}{u}\right)+\frac{\mu y}{2}\left(v-\frac{1}{v}\right)+\frac{\nu z}{2}\left(u^{2} \tau_{1}-\frac{1}{u^{2} \tau_{1}}\right)+\frac{\eta w}{2}\left(v^{2} \tau_{2}-\frac{1}{v^{2} \tau_{2}}\right)+\frac{\beta h}{2}\right. \\
\left.\left(u v \tau_{3}-\frac{1}{u v \tau_{3}}\right)\right)=\sum_{p, q, r, s, t=-\infty}^{\infty} \frac{u^{p+2 r+t} v^{q+2 s+t}}{\lambda^{h} \mu^{q} \nu^{r} \eta^{s} \beta^{t}} \tau_{1}^{r}{\tau_{2}}^{s} \tau_{3}{ }^{t} J_{p}(x) J_{q}(y) J_{r}(z) J_{s}(w) J_{t}(h) \\
\sum_{i, j=0}^{\infty} \frac{u^{i} v^{j}}{i ! j !} H_{i, j}\left(\frac{x}{2 \lambda}\left(\lambda^{2}-1\right), \frac{y}{2 \mu}\left(\mu^{2}-1\right), \frac{z \tau_{1}}{2 \nu}\left(\nu^{2}-1\right), \frac{w \tau_{2}}{2 \eta}\left(\eta^{2}-1\right), \frac{h \tau_{3}}{2 \beta}\left(\beta^{2}-1\right)\right) .
\end{gathered}
$$

Now, replacing $p$ by $l-2 r$ and $q$ by $k-2 s$ and using the relation (7) in the r.h.s. of Eq.(43), we find

$$
\begin{aligned}
& \exp \left(\frac{\lambda x}{2}\left(u-\frac{1}{u}\right)+\frac{\mu y}{2}\left(v-\frac{1}{v}\right)+\frac{\nu z}{2}\left(u^{2} \tau_{1}-\frac{1}{u^{2} \tau_{1}}\right)+\frac{\eta w}{2}\left(v^{2} \tau_{2}-\frac{1}{v^{2} \tau_{2}}\right)+\frac{\beta h}{2}\right. \\
& \left.\left(u v \tau_{3}-\frac{1}{u v \tau_{3}}\right)\right)=\sum_{l, k, t=-\infty}^{\infty} \frac{u^{l+t} v^{k+t}}{\lambda^{l} \mu^{k} \beta^{t}}\left(\frac{\lambda \mu \tau_{3}}{\beta}\right)^{t} J_{l}\left(x, z ; \frac{\lambda^{2} \tau_{1}}{\nu}\right) J_{k}\left(y, w ; \frac{\mu^{2} \tau_{2}}{\eta}\right) J_{t}(h)
\end{aligned}
$$




$$
\sum_{i, j=0}^{\infty} \frac{u^{i} v^{j}}{i ! j !} H_{i, j}\left(\frac{x}{2 \lambda}\left(\lambda^{2}-1\right), \frac{y}{2 \mu}\left(\mu^{2}-1\right), \frac{z \tau_{1}}{2 \nu}\left(\nu^{2}-1\right), \frac{w \tau_{2}}{2 \eta}\left(\eta^{2}-1\right), \frac{h \tau_{3}}{2 \beta}\left(\beta^{2}-1\right)\right) .
$$

Again, replacing $l$ by $\theta-t$ and $k$ by $\phi-t$ and using the relation (12) in the r.h.s. of Eq.(44), we get

$$
\begin{gathered}
\exp \left(\frac{\lambda x}{2}\left(u-\frac{1}{u}\right)+\frac{\mu y}{2}\left(v-\frac{1}{v}\right)+\frac{\nu z}{2}\left(u^{2} \tau_{1}-\frac{1}{u^{2} \tau_{1}}\right)+\frac{\eta w}{2}\left(v^{2} \tau_{2}-\frac{1}{v^{2} \tau_{2}}\right)+\frac{\beta h}{2}\right. \\
\left.\left(u v \tau_{3}-\frac{1}{u v \tau_{3}}\right)\right)=\sum_{\theta, \phi=-\infty}^{\infty} \sum_{i, j=0}^{\infty} \frac{u^{\theta+i} v^{\phi+j}}{\lambda^{\theta} \mu^{\phi} i ! j !} J_{\theta, \phi}\left(x, y, z, w, h ; \frac{\lambda^{2} \tau_{1}}{\nu}, \frac{\mu^{2} \tau_{2}}{\eta}, \frac{\lambda \mu \tau_{3}}{\beta}\right) \\
H_{i, j}\left(\frac{x}{2 \lambda}\left(\lambda^{2}-1\right), \frac{y}{2 \mu}\left(\mu^{2}-1\right), \frac{z \tau_{1}}{2 \nu}\left(\nu^{2}-1\right), \frac{w \tau_{2}}{2 \eta}\left(\eta^{2}-1\right), \frac{h \tau_{3}}{2 \beta}\left(\beta^{2}-1\right)\right) .
\end{gathered}
$$

Further, replacing $\theta$ by $m-i$ and $\phi$ by $n-j$ in the r.h.s. and using the definition (13) on the l.h.s. of above equation, we find

$$
\begin{aligned}
& \sum_{m, n=-\infty}^{\infty} J_{m, n}\left(\lambda x, \mu y, \nu z, \eta w, \beta h ; \tau_{1}, \tau_{2}, \tau_{3}\right) u^{m} v^{n}=\sum_{m, n=-\infty}^{\infty} u^{m} v^{n} \lambda^{-m} \mu^{-n} \\
& \sum_{i, j=0}^{\infty} \frac{\lambda^{i} \mu^{j}}{i ! j !} J_{m-i, n-j}\left(x, y, z, w, h ; \frac{\lambda^{2} \tau_{1}}{\nu}, \frac{\mu^{2} \tau_{2}}{\eta}, \frac{\lambda \mu \tau_{3}}{\beta}\right) \\
& H_{i, j}\left(\frac{x}{2 \lambda}\left(\lambda^{2}-1\right), \frac{y}{2 \mu}\left(\mu^{2}-1\right), \frac{z \tau_{1}}{2 \nu}\left(\nu^{2}-1\right), \frac{w \tau_{2}}{2 \eta}\left(\eta^{2}-1\right), \frac{h \tau_{3}}{2 \beta}\left(\beta^{2}-1\right)\right) .
\end{aligned}
$$

Finally, on equating the coefficients of like powers of $u$ and $v$ in Eq. (46), we get the assertion (41) of Theorem 2.2.

\section{Applications}

We discuss some applications of the multiplication theorems obtained in the preceding section.

I. Taking $z=\tau_{2}=0$ and replacing $\tau_{1}$ by $\tau$ in Eq. (35) and using Eq. (5), we obtain the multiplication theorem for 2V1PGBF $J_{n}(\lambda x, \mu y ; \tau)([12]$, p.145(10))

$$
J_{n}(\lambda x, \mu y ; \tau)=\lambda^{-n} \sum_{m=0}^{\infty} \frac{1}{m !} J_{n-m}\left(x, y ; \frac{\lambda^{2} \tau}{\mu}\right) H_{m}\left(\frac{x}{2}\left(\lambda^{2}-1\right), \frac{\lambda^{2} \tau y}{2 \mu}\left(\mu^{2}-1\right)\right),
$$

where $H_{n}(x, y)$ denotes the 2 VHKdFP given by Eq. (28).

II. Replacing $\tau_{1}$ by $\frac{\mu}{\lambda^{2}}$ and $\tau_{2}$ by $\frac{\nu}{\lambda^{3}}$ in Eq. (35) and using Eq. (33), we get

$$
\begin{aligned}
J_{n}\left(\lambda x, \mu y, \nu z ; \frac{\mu}{\lambda^{2}}, \frac{\nu}{\lambda^{3}}\right)= & \lambda^{-n} \sum_{m, r=0}^{\infty} \frac{1}{m ! r !(n+r) !} H_{n-m+r}\left(\frac{x}{2}, \frac{y}{2}, \frac{z}{2}\right) H_{r}\left(\frac{-x}{2}, \frac{-y}{2}, \frac{-z}{2}\right) \\
& H_{m}\left(\frac{x}{2}\left(\lambda^{2}-1\right), \frac{y}{2}\left(\mu^{2}-1\right), \frac{z}{2}\left(\nu^{2}-1\right)\right),
\end{aligned}
$$

where $J_{n}\left(x, y, z ; \tau_{1}, \tau_{2}\right)$ denotes the $3 \mathrm{~V} 2 \mathrm{PBF}$ given by Eq. (2) and $H_{n}(x, y, z)$ denotes the $3 \mathrm{VGHP}$ given by Eq. (27).

III. Taking $\tau_{1}=\tau_{2}=1$ in Eq. (35) and using Eq. (3), we obtain the multiplication theorem for 3VGBF $J_{n}(\lambda x, \mu y, \nu z)$

$$
J_{n}(\lambda x, \mu y, \nu z)=\lambda^{-n} \sum_{m=0}^{\infty} \frac{1}{m !} J_{n-m}\left(x, y, z ; \frac{\lambda^{2}}{\mu}, \frac{\lambda^{3}}{\nu}\right) H_{m}\left(\frac{x}{2}\left(\lambda^{2}-1\right), \frac{\lambda^{2} y}{2 \mu}\left(\mu^{2}-1\right), \frac{\lambda^{3} z}{2 \nu}\left(\nu^{2}-1\right)\right) .
$$

where $J_{n}(x, y, z)$ denotes the $3 \mathrm{VGBF}$ given by Eq. (4). 
IV. Taking $\tau_{1}=\tau_{2}=\tau_{3}=1$ in Eq. (41) and using Eq. (14), we get the multiplication theorem for $2 \mathrm{I} 5 \mathrm{VBF}$ $J_{m, n}(\lambda x, \mu y, \nu z, \eta w, \beta h)$

$$
\begin{array}{r}
J_{m, n}(\lambda x, \mu y, \nu z, \eta w, \beta h)=\lambda^{-m} \mu^{-n} \sum_{i, j=0}^{\infty} \frac{\lambda^{i} \mu^{j}}{i ! j !} J_{m-i, n-j}\left(x, y, z, w, h ; \frac{\lambda^{2}}{\nu}, \frac{\mu^{2}}{\eta}, \frac{\lambda \mu}{\beta}\right) \\
H_{i, j}\left(\frac{x}{2 \lambda}\left(\lambda^{2}-1\right), \frac{y}{2 \mu}\left(\mu^{2}-1\right), \frac{z}{2 \nu}\left(\nu^{2}-1\right), \frac{w}{2 \eta}\left(\eta^{2}-1\right), \frac{h}{2 \beta}\left(\beta^{2}-1\right)\right) .
\end{array}
$$

where $J_{m, n}\left(x, y, z, w, h ; \tau_{1}, \tau_{2}, \tau_{3}\right)$ denotes the $2 \mathrm{I} 5 \mathrm{~V} 3 \mathrm{PBF}$ given by Eq. (13) and $H_{i, j}(x, y, z, w, h)$ denotes the 2I5VGHP given by Eq. (30).

V. Taking $z=w=0, \tau_{3}=1$ and replacing $h$ by $z$ in Eq. (41) and using Eq. (18), we find the multiplication theorem for $2 \mathrm{I} 3 \mathrm{VBF} J_{m, n}(\lambda x, \mu y, \beta z)$

$$
J_{m, n}(\lambda x, \mu y, \beta z)=\lambda^{-m} \mu^{-n} \sum_{i, j=0}^{\infty} \frac{\lambda^{i} \mu^{j}}{i ! j !} J_{m-i, n-j}\left(x, y, z ; \frac{\lambda \mu}{\beta}\right) H_{i, j}\left(\frac{x}{2 \lambda}\left(\lambda^{2}-1\right), \frac{y}{2 \mu}\left(\mu^{2}-1\right), \frac{z}{2 \beta}\left(\beta^{2}-1\right)\right),
$$

where $J_{m, n}(x, y, z ; \tau)$ denotes the 2I3V1PBF given by Eq. (17) and $H_{i, j}(x, y, z)$ denotes the 2I3VGHP given by Eq. (31). Further, replacing $y$ and $z$ by $x$ and $\mu$ and $\beta$ by $\lambda$ in Eq. (51) and using Eq. (20), we obtain a result $([12], p .146(11))$.

VI. Replacing $\tau_{1}$ by $\frac{\nu}{\lambda^{2}}, \tau_{2}$ by $\frac{\eta}{\mu^{2}}$ and $\tau_{3}$ by $\frac{\beta}{\lambda \mu}$ in Eq. (41) and using Eq. (34), we get

$$
\begin{gathered}
J_{m, n}\left(\lambda x, \mu y, \nu z, \eta w, \beta h ; \frac{\nu}{\lambda^{2}}, \frac{\eta}{\mu^{2}}, \frac{\beta}{\lambda \mu}\right)=\lambda^{-m} \mu^{-n} \sum_{i, j, r, s=0}^{\infty} \frac{\lambda^{i} \mu^{j}}{i ! j ! r ! s !(m+r) !(n+s) !} \\
H_{m-i+r, n-j+s}\left(\frac{x}{2}, \frac{y}{2}, \frac{z}{2}, \frac{w}{2}, \frac{h}{2}\right) H_{r, s}\left(\frac{-x}{2}, \frac{-y}{2}, \frac{-z}{2}, \frac{-w}{2}, \frac{-h}{2}\right) \\
H_{i, j}\left(\frac{x}{2 \lambda}\left(\lambda^{2}-1\right), \frac{y}{2 \mu}\left(\mu^{2}-1\right), \frac{z \tau_{1}}{2 \nu}\left(\nu^{2}-1\right), \frac{w \tau_{2}}{2 \eta}\left(\eta^{2}-1\right), \frac{h \tau_{3}}{2 \beta}\left(\beta^{2}-1\right)\right) .
\end{gathered}
$$

VII. Taking $y=w=h=0, \tau_{1}=1$ and replacing $z$ by $y$ in Eq. (41) and using Eq. (23), we get the multiplication theorem for $2 \mathrm{VBF} J_{m}(\lambda x, \nu y)$

$$
J_{m}(\lambda x, \nu y)=\lambda^{-m} \sum_{i=0}^{\infty} \frac{\lambda^{i}}{i !} J_{m-i}\left(x, y ; \frac{\lambda^{2}}{\nu}\right) H_{i}\left(\frac{x}{2 \lambda}\left(\lambda^{2}-1\right), \frac{y}{2 \nu}\left(\nu^{2}-1\right)\right),
$$

or, taking $x=z=h=0, \tau_{2}=1$ and replacing $y$ by $x$ and $w$ by $y$ in Eq. (41) and using Eq. (24), we get

$$
J_{n}(\mu x, \eta y)=\mu^{-n} \sum_{j=0}^{\infty} \frac{\mu^{j}}{j !} J_{n-j}\left(x, y ; \frac{\mu^{2}}{\eta}\right) H_{j}\left(\frac{x}{2 \mu}\left(\mu^{2}-1\right), \frac{y}{2 \eta}\left(\eta^{2}-1\right)\right),
$$

where $J_{n}(x, y ; \tau)$ denotes the $2 \mathrm{~V} 1 \mathrm{PBF}$ given by Eq. (6) and $J_{n}(x, y)$ denotes the 2VGBF given by Eq. (9).

VIII. Taking $y=z=w=h=0$ in Eq. (41) and using Eqs. (25) and (29), we find the multiplication theorem for BF $J_{m}(\lambda x)([12], p .150(25))$

$$
J_{m}(\lambda x)=\lambda^{-m} \sum_{i=0}^{\infty} \frac{1}{i !}\left(x\left(\lambda^{2}-1\right)\right)^{i} J_{m-i}(x),
$$

or, taking $x=z=w=h=0$ and replacing $y$ by $x$ in Eq. (41) and using Eqs. (26) and (29), we get

$$
J_{n}(\mu x)=\mu^{-n} \sum_{j=0}^{\infty} \frac{1}{j !}\left(x\left(\mu^{2}-1\right)\right)^{j} J_{n-j}(x),
$$

where $J_{n}(x)$ denotes the ordinary Bessel functions. 


\section{Conclusion}

In section 2 , we have derive multiplication theorems for the $3 \mathrm{~V} 2 \mathrm{PBF} J_{n}\left(\lambda x, \mu y, \nu z ; \tau_{1}, \tau_{2}\right)$ and $2 \mathrm{I} 5 \mathrm{~V} 3 \mathrm{PBF}$ $J_{m, n}\left(\lambda x, \mu y, \nu z, \eta w, \beta h ; \tau_{1}, \tau_{2}, \tau_{3}\right)$ using the generating function method. We remark that this process can be extended to establish multiplication theorem for the N-IBF $J_{m_{1}, m_{2}, \ldots . ., m_{N}}(\lambda x)$. First, we recall the N-IBF $J_{m_{1}, m_{2}, \ldots . ., m_{N}}(x)$ defined by the generating function $[6]$

$$
\sum_{m_{j}=-\infty}^{\infty} J_{m_{1}, \ldots, m_{N}}(x) \prod_{j=1}^{N} u_{j}^{m_{j}}=\exp \left(\frac{x}{2}\left(\sum_{j=1}^{N}\left(u_{j}-\frac{1}{u_{j}}\right)+\left(\prod_{j=1}^{N} u_{j}-\frac{1}{\prod_{j=1}^{N} u_{j}}\right)\right)\right),
$$

with the following series

$$
J_{m_{1}, \ldots, m_{N}}(x)=\sum_{l=-\infty}^{\infty}\left(\prod_{j=1}^{N} J_{m_{j}-l}(x)\right) J_{l}(x) .
$$

Also, we can define the N-index 1-variable 1-parameter Bessel functions (N-I1V1PBF) $J_{m_{1}, \ldots, m_{N}}(x ; \tau)$ by means of the series

$$
J_{m_{1}, \ldots, m_{N}}(x ; \tau)=\sum_{l=-\infty}^{\infty} \tau^{(N-1) l}\left(\prod_{j=1}^{N} J_{m_{j}-l}(x)\right) J_{l}(x) .
$$

Now, we start with the identity

$$
\begin{array}{r}
\exp \left(\frac{\lambda x}{2}\left(\sum_{j=1}^{N}\left(u_{j}-\frac{1}{u_{j}}\right)+\left(\prod_{j=1}^{N} u_{j}-\frac{1}{\prod_{j=1}^{N} u_{j}}\right)\right)\right)=\exp \left(\frac{x}{2}\left(\sum_{j=1}^{N}\left(\frac{u_{j}}{\lambda}-\frac{\lambda}{u_{j}}\right)\right)\right) \\
\exp \left(\frac{x}{2}\left(\prod_{j=1}^{N} \frac{u_{j}}{\lambda}-\prod_{j=1}^{N} \frac{\lambda}{u_{j}}\right)\right) \exp \left(\frac{x}{2}\left(\frac{\lambda^{2}-1}{\lambda}\right)\left(\sum_{j=1}^{N} u_{j}+\prod_{j=1}^{N} u_{j}\right)\right),
\end{array}
$$

which on using the definition (11), on the r.h.s. becomes

$$
\begin{aligned}
& \exp \left(\frac{\lambda x}{2}\left(\sum_{j=1}^{N}\left(u_{j}-\frac{1}{u_{j}}\right)+\left(\prod_{j=1}^{N} u_{j}-\frac{1}{\prod_{j=1}^{N} u_{j}}\right)\right)\right) \\
& =\sum_{p_{j}, r_{j}, q=-\infty}^{\infty} \frac{\prod_{j=1}^{N} u_{j} p_{j}+r_{j}+q}{\lambda^{p_{j}+q} r_{1} !, r_{2} !, \ldots ., r_{N} !} J_{p_{1}}(x) J_{p_{2}}(x) \ldots . . J_{p_{N}}(x) J_{q}(x) H_{r_{1}, r_{2}, \ldots ., r_{N}}\left(x\left(\frac{\lambda^{2}-1}{\lambda}\right)\right),
\end{aligned}
$$

where

$$
H_{r_{1}, r_{2}, \ldots, r_{N}}(x)=\sum_{k=0}^{\min \left(r_{1}, r_{2}, \ldots, r_{N}\right)}\left(\frac{x}{2}\right)^{\sum_{j=1}^{N} r_{j}-(N-1) k} \frac{r_{1} !, r_{2} !, r_{3} !, \ldots \ldots, r_{N} !}{k !\left(r_{1}-k\right) !\left(r_{2}-k\right) ! \ldots .\left(r_{N}-k\right) !} .
$$

Now, replacing $p_{j}$ by $s_{j}-q$ and using the relation (59) in the r.h.s. of Eq.(61), we find

$$
\begin{gathered}
\exp \left(\frac{\lambda x}{2}\left(\sum_{j=1}^{N}\left(u_{j}-\frac{1}{u_{j}}\right)+\left(\prod_{j=1}^{N} u_{j}-\frac{1}{\prod_{j=1}^{N} u_{j}}\right)\right)\right)=\sum_{s_{j}, r_{j}=-\infty}^{\infty} \frac{\prod_{j=1}^{N} u_{j}^{s_{j}+r_{j}}}{\lambda^{s_{i}} r_{1} !, r_{2} !, \ldots ., r_{N} !} J_{s_{1}, s_{2}, \ldots, s_{N}}(x ; \lambda) \\
H_{r_{1}, r_{2}, \ldots, r_{N}}\left(x\left(\frac{\lambda^{2}-1}{\lambda}\right)\right) .
\end{gathered}
$$

Further, replacing $s_{j}$ by $m_{j}-r_{j}$ in the r.h.s. and using definition (57) on the l.h.s. of above equation, we find

$$
\begin{aligned}
\sum_{m_{j}=-\infty}^{\infty} J_{m_{1}, \ldots ., m_{N}}(\lambda x) \prod_{j=1}^{N} u_{j}^{m_{j}}= & \sum_{m_{j}, r_{j}=-\infty}^{\infty} \frac{\prod_{j=1}^{N} u_{j}^{m_{j}}}{\lambda^{m_{j}-r_{j}} r_{1} !, r_{2} !, \ldots ., r_{N} !} J_{m_{1}-r_{1}, m_{2}-r_{2}, \ldots ., m_{N}-r_{N}}(x ; \lambda) \\
& H_{r_{1}, r_{2}, \ldots ., r_{N}}\left(x\left(\frac{\lambda^{2}-1}{\lambda}\right)\right) .
\end{aligned}
$$


Finally, on equating the coefficients of like powers of $\prod_{j=1}^{N} u_{j}{ }^{m_{j}}$ in Eq. (63), we get multiplication theorem for the N-IBF $J_{m_{1}, m_{2}, \ldots . ., m_{N}}(\lambda x)$ as follows

$$
J_{m_{1}, \ldots, m_{N}}(\lambda x)=\lambda^{-m_{1}-m_{2} \ldots-m_{N}} \sum_{r_{j}=-\infty}^{\infty} \frac{\lambda^{r_{1}+r_{2}+\ldots .+r_{N}}}{r_{1} !, r_{2} !, \ldots, r_{N} !} J_{m_{1}-r_{1}, m_{2}-r_{2}, \ldots, m_{N}-r_{N}}(x ; \lambda) H_{r_{1}, r_{2}, \ldots, r_{N}}\left(x\left(\frac{\lambda^{2}-1}{\lambda}\right)\right) .
$$

The theory of BF is rich and wide and certainly provides an inexhaustible field of research. A large number of functions are recognized as belonging to the BF family. The above multiplication theorem can be viewed as a multi-index extension of multiplication theorem (3.9) for the BF $J_{m}(x)$. Exploring the possibility of using the method outlined here to derive the multiplication theorems for other special functions is a further research problems.

\section{References}

[1] C. Chiccoli, G. Dattoli, S. Lorenzutta, G. Maino and A. Torre, Theory of one parameter generalized Bessel functions, Monograph, Gruppo Nazionale Informatica Mathematica CNR, Rome, 1992.

[2] G. Dattoli, C. Chiccoli, S. Lorenzutta, G. Maino, M. Richetta and A. Torre,. Generating functions of multi-variable generalized Bessel functions and Jacobi-elliptic functions, Journal of Mathematical Physics, 33(1)(1992), pp. 25-36.

[3] G. Dattoli, C. Chiccoli, S. Lorenzutta, G. Maino and A. Torre, Generalized Bessel functions and generalized Hermite polynomials, Journal of Mathematical Analysis and Applications, 178(1993), pp. 509-516.

[4] G. Dattoli, L. Giannessi, L. Mezi and A. Torre, Theory of generalized Bessel functions, Nuovo Cimento B, 105(1990), pp. 327-343.

[5] G. Dattoli, S. Lorenzutta, G. Maino and A. Torre, Generalized forms of Bessel functions and Hermite polynomials, Annals Nuemrical Mathematics, 2(1995), pp. 221-232.

[6] G. Dattoli, S. Lorenzutta, G. Maino and A. Torre, Theory of multiindex mulivariable Bessel functions and Hermite polynomials, Mathematiche(Catania), 52(1997), Fasc1, pp. 179-197.

[7] G. Dattoli, S. Lorenzutta, G. Maino, A. Torre, G. Voykov and C. Chiccoli, Theory of two index Bessel functions and applications to physical problems, Journal of Mathematical Physics, 35(7)(1994), pp. 3636-3649.

[8] G. Dattoli, M. Migliorati and H. M. Srivastava, Some families of generating Functions for the Bessel and related functions, Geogian Mathematical Journal, 11(2)(2004), pp. 219-228.

[9] G. Dattoli, A. Renieri and A. Torre, Lectures on the free Electron Laser Theory and Related Topics, Singapore, 1993.

[10] G. Dattoli and A. Torre, Theory and Applications of Generalized Bessel Functions, ARACNE, Rome, Italy, 1996.

[11] G. Dattoli, A. Torre, S. Lorenzutta, G. Maino and C. Chiccoli, Theory of generalized Bessel functions II, Nuovo Cimento B, 106(1991), pp. 21-32.

[12] L. Galue, H. G. Khajah and S. L. Kalla, Multiplications theorem for generalized and double-index Bessel functions, Journal of Computational and Applied Mathematics, 118 (2000), pp. 143-150.

[13] E. D. Rainville, Special Functions, Macmillan, New York, 1960.

[14] Subuhi Khan and G. Yasmin, Generalized Bessel functions and Lie algebra representation, Mathematical Physics Analysis and Geometry, 8 (2005), pp. 299-313. 